\title{
Video Display Terminal Device
}

National Cancer Institute

\section{Source}

National Cancer Institute. Video Display Terminal Device. NCI Thesaurus. Code C50264.

A device incorporating a cathode ray tube, a keyboard and a computer connection designed to render data from a computer system. 